\title{
SEPSIS. Educational and Best Practice Frontiers. Beyond the Boundaries of Fatality, Enhancing Clinical Skills and Precision Medicine
}

This article was published in the following Dove Press journal:

Therapeutics and Clinical Risk Management

\section{Guglielmo Trovato}

The European Medical Association (EMA) and the School of Medicine, State University of Catania, Catania, Italy

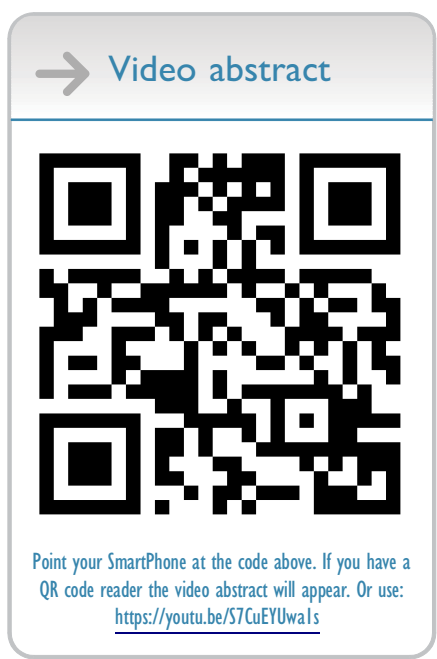

Correspondence: Guglielmo Trovato Email trovato.eu@gmail.com

\begin{abstract}
Dissemination and exploitation of knowledge regarding affordable clinical skills and innovative precision medicine, two current topics in active development in medicine, may contribute to improve also sepsis management. Sepsis is a life-threatening organ dysfunction due to a dysregulated host response to infection. Sepsis is strongly related to all body organs or to systemic diseases and to the quality of the best-practice in use, which is particularly critical in surgical or intervention techniques. Trauma, surgical and mini-invasive procedures, vascular or endoscopic interventions, otolaryngology, obstetrics-gynecological and urological procedures, malnutrition, dental, skin, chronic liver, kidney and respiratory disease are frequently involved. Accordingly, apart from the clinical risk analysis and management of the process of care, the actual factors that may be easily neglected are the techniques used, the personal skills of the health professionals and the quality of the equipment. The quest for biomarkers consistent with the unmet needs of medical doctors and of their patient and the efforts for overcoming bacterial antibiotic resistances are currently the main foci of medical research. In addition, in this regard, research and innovation would benefit from greater knowledge, skills and use of bioinformatics and omics. The caveats related to in-silico approaches must be flagged: algorithms may equally warrant scientific innovations or hide the lack of them; a patient is more than a set of covariates. Epidemiology and prevention includes all the actions suitable for achieving an adequate hygiene and immunization of populations and for safer hospital policies and procedures during Patients' stays. In any subset, the most unresolved critical point in sepsis is a timely diagnosis. This is impaired by low degrees of suspicion for the possibility of emerging sepsis, by the shortage of use of the simplest microbiological testing but, equally or more, by the insufficient diffusion of non-invasive imaging skills suitable to detect and monitor the emerging sites and sources of infection. In primary care, in emergency facilities, in hospital wards and in intensive care units, inclusion of appropriate knowledge, skills, expertise and imaging equipment must be extended as much as possible. The low cost of UltraSound machines and of increasing bioinformatics literacy by e-learning, makes such investments affordable even in limited-resources contexts. Frontier educational and best practice intervention enhancing affordable clinical skills and innovative precision medicine may lead beyond the boundaries of fatal outcomes in sepsis.
\end{abstract}

Keywords: sepsis, bioinformatics, ultrasound, e-learning, MOOC, genomics, research models

\section{Background}

Sepsis is a life-threatening organ dysfunction due to a dysregulated host response to infection. ${ }^{1}$ Organ dysfunction is defined as an increase of 2 points or more in the Sequential Organ Failure Assessment (SOFA) score, aimed at detecting and monitoring with prompt bedside analysis in patients who have suspected or documented 
infection. ${ }^{2}$ Sepsis is strongly related to all body organs or to systemic diseases and to the quality of the best-practice in use, which is particularly critical in surgical or intervention techniques. Trauma, surgical and mini-invasive procedures, vascular or endoscopic interventions, otolaryngology, obstetrics-gynecological and urological procedures, malnutrition, dental, skin, chronic liver, kidney and respiratory disease are frequently involved as the genesis of disseminated infections. From this point of view, apart from the clinical risk analysis and management of the entire process of care, the techniques used, and the personal skills of the health professionals, with the quality of the equipment available, are significant actual factors that may be easily overlooked. ${ }^{3}$ Multiple organ dysfunction syndrome (MODS) is characterized by progressive organ dysfunction in a severely ill patient, with failure to maintain homeostasis without intervention. Sepsis, which may be an overlapping condition of other non-infectious conditions, such as pancreatic disease, may cause a great number of organ failures, with a high mortality risk. Such events are usually associated with respiratory failure requiring mechanical ventilation. The development of new scoring systems to risk-stratify emergency departmentsuspected sepsis are continuously increasing the awareness and the effectiveness of intervention. ${ }^{4}$ Dissemination and exploitation of knowledge regarding affordable clinical skills and innovative precision medicine, two current topics in active development in medicine, may contribute to improve also sepsis management.

\section{Challenges}

Professional education and training in sepsis is far from adequate. Most clinical practitioners outside intensive care medicine are unfamiliar with sepsis, even emergency physicians. Lack of sepsis education projects is a primary challenge in sepsis management. Currently, the true burden of disease arising from sepsis remains unknown, mostly because estimates are based on data referring to hospitaltreated sepsis in high-income countries. Ensuring greater awareness on the part of both the public and health care workers is a crucial step in reducing the global burden of sepsis. ${ }^{3}$ National awareness sepsis programs are few and far between, insufficiently wide-ranging and attractive for the potential audience. Overall, we are not adequately teaching all health care workers both to recognize sepsis and to understand it as a true time-critical medical emergency. International actions and projects are, seemingly, sparse and insufficiently supported and funded. The key role of the schools of medicine and of other health professions, including psychology, is currently not adequately taken into account, and focused programs to enhance knowledge and skills in sepsis recognition and management are needed worldwide. Sepsis 3.0 definition and diagnostic criteria ${ }^{2}$ based on widely used laboratory findings, vital signs, and clinical intervention (qSOFA and SOFA scores), did not include novel biomarkers or microbiological results. ${ }^{5}$ Adding bioinformatics and omics to sepsis diagnosis maybe a challenge, and their value for improving sepsis outcomes is still unclear. ${ }^{6,7}$ Applied bioinformatics and omics in clinical practice of sepsis are also limited by financial conditions. ${ }^{8,9}$ According to the Iceberg Model of Health and Disease, what we see of illness and health, and what we know as events and deaths due to sepsis is only the tip of a submerged mass (Figure 1). Looking below the surface, much like icebergs that reveal only about one-tenth of their mass above the water and the other nine-tenths remains submerged, we can see that most diagnoses of sepsis are hidden in the official statistics. This is due to several causes, including inadequate public literacy without awareness of the sepsis concept. Therefore, official reports of the cause of illness or death refer only to the most likely definition of its causative infection or factor and not as "sepsis" itself.

The use of appropriate laboratory tests for confirmation and definition of the underlying pathogenesis, and the prompt use of effective antibiotics are pillars of current sepsis management. Furthermore, the wider use of biomarkers for speeding diagnosis has already achieved some advantage providing earlier information, while waiting for the results of microbiological cultures and identification. ${ }^{6,8}$ Nonetheless, current biomarkers may be quite insensitive and excessively generic for such purposes. In addition, their use for monitoring persistence of the critical phase may be unreliable. The quest for biomarkers consistent with the unmet needs of medical doctors and of their patients and the efforts towards overcoming bacterial antibiotic resistances are currently the main focus of medical research. Also in this regard, research and innovation would benefit from greater knowledge, skills and use of bioinformatics, epigenetics ${ }^{9}$ and new biomarkers, such as presepsin. ${ }^{10}$

\section{Ideas and Available Human Resources}

Medical doctors, with their different and complementary background and expertise, share the core commitment of direct care of their patients. Accordingly, also sepsis, as 


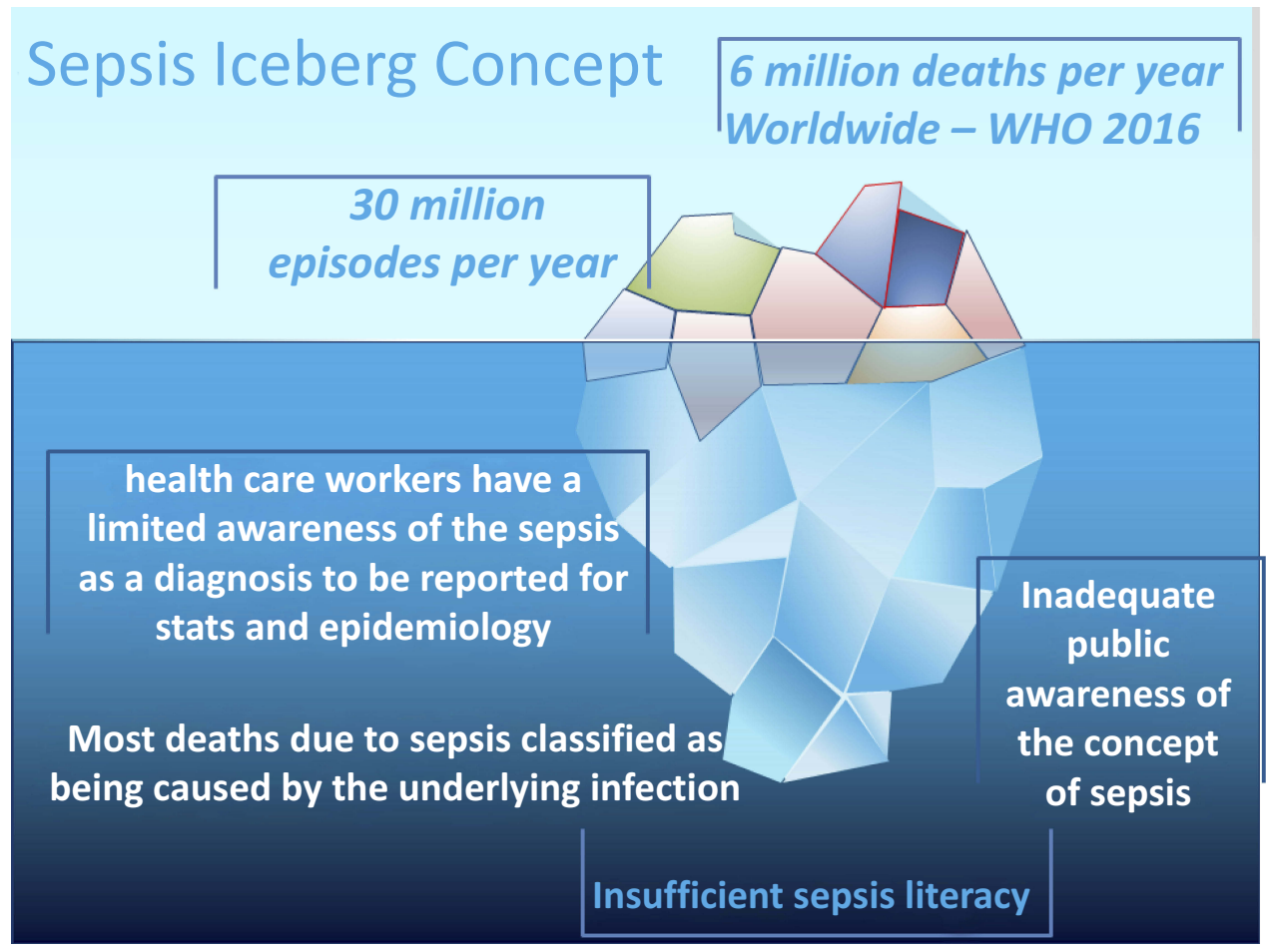

Figure I According to the Iceberg model of health and disease, what we see of illness and health, and what we know as events and deaths due to sepsis is only the tip of an iceberg. Looking below the surface, much alike icebergs that reveal only about one-tenth of their mass above the water and the other nine-tenths remains submerged, we can see that most diagnosis of sepsis are hidden in the official statistics. This is due to several causes, including inadequate public literacy without awareness of the sepsis concept. Therefore, official reports of the cause of illness or death refer only to the most likely definition of its causative infection or factor and not as "sepsis" itself.

a topic and as a challenge, must integrate inputs of different concepts, contexts and approaches of professionals directly involved in these tasks.

The empowerment of appropriate knowledge and skills of medical doctors must be warranted, facilitated and allowed, addressing those competencies and human resource profiles that are most discriminant, both in limited resource facilities and in advanced health systems. ${ }^{11}$ Best practice is not only a matter of management of clinical risks and processes, but equally, if not more so, a matter of robust clinical and scientific involvement of medical doctors when faced with sepsis. Sepsis includes most overlapping domains of clinical medicine and pathology. The demand for increasing and improving the quality of the doctor-patient relationship, which has been and remains a keystone of care, calls for a more detailed and comprehensive analysis. Such a relationship is the medium in which data are gathered, diagnoses and plans are made, compliance is accomplished, and healing patient activation and support are provided. ${ }^{12}$

\section{New Frontiers}

Research and innovation should take advantage of greater knowledge and skills in bioinformatics and "-omics", also using as biomarkers breath gases by novel non-invasive monitoring. The increase of basic and translational literacy and competence of European health professionals in bioinformatics is the valuable commitment of some European action. ${ }^{13}$ The management and interpretation of big data, not only in genetics and in -omics, ${ }^{14}$ imply having in mind the caveats related to in silico approaches: algorithms may equally warrant scientific innovations or hide the lack of it, since a patient is more than a set of covariates. ${ }^{15}$ The occurrence of antibiotic-resistance, which is one of the major concerns in the debate on sepsis, may receive answers from pharmacogenomics and computational biology approaches, ${ }^{16}$ even if currently limited experience is available in this regard.

\section{Advice and Sustainable Actions}

Apart from any innovative scenario, the possible forthcoming actions of health institutions and associations can be itemized by the following inter-related comments and suggestions, focused on achieving more performant clinical training and best practice, visually summarized in Figure 2.

1) Epidemiology and prevention include all the actions suitable for adequate hygiene and immunization of all 


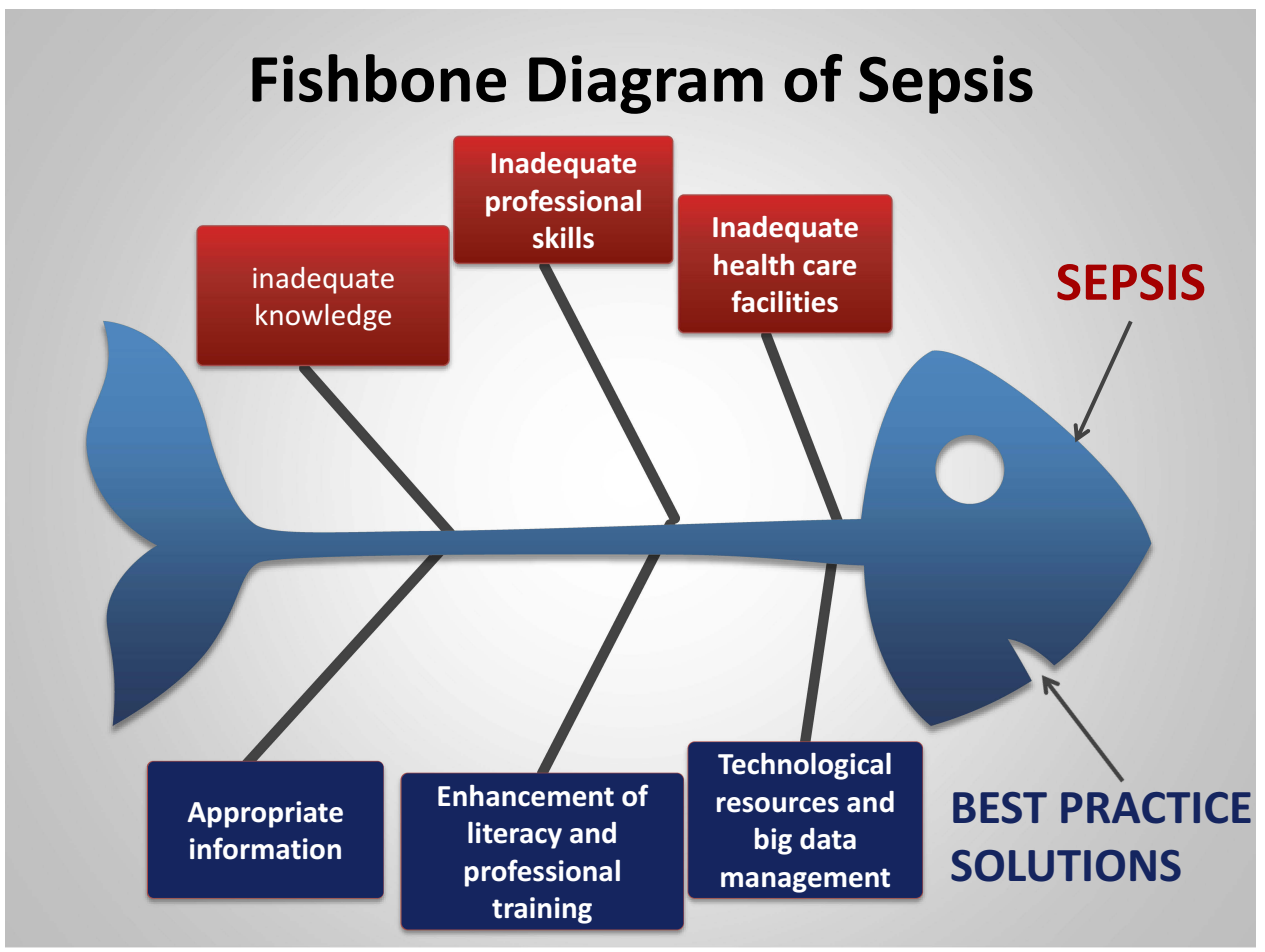

Figure 2 This "fishbone" diagram may help to identify some possible causes of quality defects in the detection, prevention and management of sepsis as a medical problem. The root causes analysis (RCA) of faults \& problems is used also in medicine, i.e. in the domains of health and safety. The RCA in diagnosis, epidemiology (e.g. to identify the source of an infectious disease), environmental science, accident analysis and occupational safety may help in sorting ideas into useful categories by a visual representation. Looking at causes and effect by a visual concept representation may help addressing to a suitable solution. The outcome or the proposed solution are displayed at the mouth of the fish. Accordingly, inadequate knowledge, skills and facility may lead to delayed detection and management of sepsis (above in the figure). Conversely, (below in the figure), increased resources dedicated to focused information and dissemination, to big-data management with sustainable technology solutions, and to an appropriate enhancement of educational and work-force profiles, are the opposite factors, leading to favorable solutions of the problem that we wish to analyze and manage by the proposed intervention.

populations and for safer procedural pathways during any hospital stay. This is only in part dependent on the financial resources of the individual patient or of the different national healthcare systems, and can be enhanced by comprehensive health literacy increase interventions, with articulated dissemination strategies. For sepsis, prevention and awareness are the most important topics in several previous and current campaigns.

2) The most unresolved critical points in sepsis are early and timely diagnosis; both are currently impaired by low degrees of suspicion for the possibility of emerging sepsis. One cause is the lack of use of the simplest microbiological testing but, equally or even more influential is the insufficient diffusion of non-invasive imaging skills suitable for detecting and monitoring the emerging sites and sources of infection. This is true, for instance, for pneumonia, ${ }^{17}$ urological infections, retroperitoneal abscesses, endocarditis and soft-tissue and vasculitic infections. ${ }^{18}$

3) In primary care, in emergency facilities, in hospital wards and in intensive care units the inclusion of appropriate knowledge, skills, expertise and bedside imaging equipment must be extended as much as possible. The very low cost of ultrasound machines makes this investment affordable even in a limited-resource context. The counterpart of this effective development is the need for providing high quality and lasting educational support in point-of-care diagnostic and intervention ultrasound procedures, including echocardiography.

4) Lasting training is necessary for gaining the essential expertise for uniform and reliable clinical practice using ultrasound machines. This allows frequent checking and monitoring of patients and, when needed, safer and precise interventional procedures targeted at biopsies or tapping or draining fluid for microscopy or microbiological assays. ${ }^{19}$ Individualized management strategies matched to a patient's molecular and biochemical profile include improved diagnostic techniques and pharmacological agents capable of modifying the disease process. "While waiting for this goal to be achieved, improved basic care driven by education and quality-improvement programmes offer the best hope of increasing favorable outcomes". ${ }^{20}$ In most intensive care contexts, including liver transplantation, sepsis can be flagged not only by 
the future achievements of bioinformatics or omics techniques, ${ }^{21,22}$ as in cancer, ${ }^{23,24}$ in liver and digestive disease $^{25}$ and in environmental and infection-related disease ${ }^{26}$ but also by point-of-care direct imaging and insight for the prevention of the possible emerging source of dissemination. ${ }^{27}$

A realistic perception of disease is the basis for a strong agreement and alliance of doctors and patients against diseases that have many hidden effects. This is a key component for better coping with post-sepsis psychological and social consequences and for reducing the area of misunderstanding between health professionals and patients. ${ }^{27}$ Support of patients with such severe diseases should not be only "consolatory", because such interventions may unmask the real needs and the quest for most appropriate potentially available expertise. ${ }^{28}$ Standard sepsis education projects for clinical practitioners should be optimally established and well performed. ${ }^{3}$ The awareness and early recognition of sepsis sources in all clinical practice subsets is the foundation of successful management of sepsis also by bioinformatics and precision medicine. ${ }^{29-34}$ Increasing bioinformatics literacy by e-learning is a well established strategy. ${ }^{35} \mathrm{~A}$ key point is that the low cost of UltraSound machines makes such investments affordable even in limited-resources settings. ${ }^{11,34-39}$ The rationale behind this last suggestion may not be obvious, and a few lines of explanation are needed. Spontaneous or iatrogenic abscess or fluid cavities are frequently the sources of bacteria in sepsis, which infect the endocardium and cause vegetation on cardiac valves. Medical doctors trained in abdominal and lung ultrasound are capable of investigating the heart valves for endocarditis by echocardiography, allowing earlier and timely diagnosis useful for preventing or ending a septic process. Moreover, medical doctors that find and show the source of sepsis by point of care imaging provide their patients with direct and visible information, also showing and sharing such aspects by the frequent monitoring of the lesions.

\section{Conclusion}

A diagram of root causes analysis (RCA) of faults \& problems (Figure 2) visually displays possible causes of quality defects in the detection, prevention and management of sepsis as a medical problem. Such models are used also in medicine, i.e. in the domains of health and safety. ${ }^{40}$ Root Causes Analysis in diagnosis, epidemiology (e.g. to identify the source of an infectious disease), environmental science, accident analysis and occupational safety may help in sorting ideas into useful categories by a visual representation. Looking at causes and effect through a visual concept representation may help in addressing a suitable solution. Inadequate knowledge, skills and facility may lead to delayed detection and management of sepsis: conversely, increased resources dedicated to focused information and dissemination, to big-data management with sustainable technology solutions, and to an appropriate enhancement of educational and work-force profiles may lead to favorable solutions and effective intervention. Enhancing affordable clinical skills and innovative precision medicine will lead us beyond the boundaries of fatality in sepsis, along roads facilitated by frontier education and bestpractice intervention.

\section{Acknowledgments}

A speech dealing with the topics of this paper was presented in a meeting at the European Parliament on behalf of EMA and of Health First Europe -HFE-. This last is a non-profit, noncommercial alliance of patients, healthcare workers, academics and healthcare experts and the medical technology industry. Its mission is to ensure that equitable access to modern, innovative and reliable medical technology and healthcare is regarded as a vital investment in the future of Europe.

The valuable help and suggestions for the drafting of this manuscript provided by Simon Taylor-Robinson, MD, was greatly appreciated. This article is also an educational tool of BioS Project, an Erasmus Plus action which developed a Multilanguage e-learning course in Bioinformatics and computational biology, disseminated also through social media: Twitter, Fecebook and LinkedIn. The BioS Course is already available and the Project is in active development with multiple Partners, including EMA. The European Commission support for the production of this publication does not constitute an endorsement of its contents, which reflects the views only of the author, and the Commission cannot be held responsible for any use which may be made of the information contained therein.

\section{Disclosure}

The author reports no conflicts of interest in this work.

\section{References}

1. Singer M, Deutschman CS, Seymour CW, et al. The third international consensus definitions for sepsis and septic shock (sepsis-3). JAMA. 2016;315:801-810. doi:10.1001/jama.2016.0287

2. Shankar-Hari M, Phillips GS, Levy ML, et al. Sepsis definitions task force. developing a new definition and assessing new clinical criteria for septic shock: for the third international consensus definitions for sepsis and septic shock (sepsis-3). JAMA. 2016;315:775-787. doi:10.1001/jama.2016.0289 
3. Reinhart K, Daniels R, Kissoon N, Machado FR, Schachter RD, Finfer S. Recognizing sepsis as a global health priority - a WHO resolution. $N$ Engl $J$ Med. 2017;377:414-417. doi:10.1056/ NEJMp1707170

4. Sivayoham N, Blake LA, Tharimoopantavida SE, et al. The REDS score: a new scoring system to risk-stratify emergency department suspected sepsis: a derivation and validation study. BMJ Open. 2019;26(9):e030922. doi:10.1136/bmjopen-2019-030922

5. Itenov TS, Murray DD, Jensen JUS. Sepsis: personalized medicine utilizing 'omic' technologies - a paradigm shift? Healthcare (Basel). 2018;6:E111. doi:10.3390/healthcare6030111

6. Bruse N, Leijte GP, Pickkers P, Kox M. New frontiers in precision medicine for sepsis-induced immunoparalysis. Expert Rev Clin Immunol. 2019;15:251-263. doi:10.1080/1744666X.2019.1562336

7. Murray DD, Itenov TS, Sivapalan P, et al. Biomarkers of acute lung injury the individualized approach: for phenotyping, risk stratification and treatment surveillance. J Clin Med. 2019;8:E1163. doi:10.3390/ jem8081163

8. Nunez Lopez O, Cambiaso-Daniel J, Branski LK, Norbury WB, Herndon DN. Predicting and managing sepsis in burn patients: current perspectives. Ther Clin Risk Manag. 2017;13:1107-1117. doi:10.2147/TCRM.S119938

9. Cross D, Drury R, Hill J, Pollard AJ. Epigenetics in sepsis: understanding its role in endothelial dysfunction, immunosuppression, and potential therapeutics. Front Immunol. 2019;10:1363. doi:10.3389/ fimmu.2019.01363

10. Zhu Y, Li X, Guo P, Chen Y, Li J, Tao T. The accuracy assessment of presepsin (sCD14-ST) for mortality prediction in adult patients with sepsis and a head-to-head comparison to PCT: a meta-analysis. Ther Clin Risk Manag. 2019;13(15):741-753. doi:10.2147/TCRM.S198735

11. Trovato FM, Catalano D. Diagnosis of pneumonia by lung ultrasound in children and limited resources subsets: a valuable medical breakthrough. Chest. 2016;150:258-260. doi:10.1016/j.chest.2016.04.032

12. Rosendahl J, Brunkhorst FM, Jaenichen D, Strauss B. Physical and mental health in patients and spouses after intensive care of severe sepsis: a dyadic perspective on long-term sequelae testing the actor-partner interdependence model. Crit Care Med. 2013;41:69-75. doi:10.1097/CCM.0b013e31826766b0

13. Trovato GM, Brischetto D, Dorn LE, Costigliola V. The BiosProject: an Erasmus+ European action for enhancing bioinformatics and computational biology knowledge, skills and literacy. FASEB $J$. 2019;33(1supplement):617.29.

14. Atreya MR, Wong HR. Precision medicine in pediatric sepsis. Curr Opin Pediatr. 2019;31:322-327. doi:10.1097/MOP.0000000000000753

15. Janssens ACJW. Proprietary algorithms for polygenic risk: protecting scientific innovation or hiding the lack of it? Genes (Basel). 2019;10 (6):E448. doi:10.3390/genes 10060448

16. Montassier E, Al-Ghalith GA, Ward T, et al. Pretreatment gut microbiome predicts chemotherapy-related bloodstream infection. Genome Med. 2016;8:49. doi:10.1186/s13073-016-0301-4

17. Interrigi MC, Trovato FM, Catalano D, Trovato GM. Emergency thoracic ultrasound and clinical risk management. Ther Clin Risk Manag. 2017;9:151-160. doi:10.2147/TCRM.S126770

18. Trovato GM, Sperandeo M, Catalano D. Thoracic ultrasound guidance for access to pleural, peritoneal, and pericardial space. Chest. 2013;144:1735-1736. doi:10.1378/chest.13-1475

19. Cecconi M, Evans L, Levy M, Rhodes A. Sepsis and septic shock. Lancet. 2018;392:75-87. doi:10.1016/S0140-6736(18)30696-2

20. Bajaj JS, Kakiyama G, Cox IJ, et al. Alterations in gut microbial function following liver transplant. Liver Transpl. 2018;24:752-761. doi:10.1002/lt.25046

21. Liang Y, Zhang C, Dai DQ. Identification of differentially expressed genes regulated by methylation in colon cancer based on bioinformatics analysis. World $J$ Gastroenterol. 2019;25:3392-3407. doi:10.3748/wjg.v25.i26.3392
22. Dhanasekaran R, Nault JC, Roberts LR, Zucman-Rossi J. Genomic medicine and implications for hepatocellular carcinoma prevention and therapy. Gastroenterology. 2019;156:492-509. doi:10.1053/j. gastro.2018.11.001

23. Wooden B, Goossens N, Hoshida Y, Friedman SL. Using big data to discover diagnostics and therapeutics for gastrointestinal and liver diseases. Gastroenterology. 2017;152:53-67.e3. doi:10.1053/j.gastro. 2016.09.065

24. Chang L, Di Lorenzo C, Farrugia G, et al. Functional bowel disorders: a roadmap to guide the next generation of research. Gastroenterology. 2018;154:723-735. doi:10.1053/j.gastro.2017.12.010

25. Cavalli G, Heard E. Advances in epigenetics link genetics to the environment and disease. Nature. 2019;571:489-499. doi:10.1038/ s41586-019-1411-0

26. Trovato FM, Rabinowich L, McPhail MJW. Update on the management of acute liver failure. Curr Opin Crit Care. 2019;25:157-164. doi:10.1097/MCC.0000000000000583

27. König C, Matt B, Kortgen A, Turnbull AE, Hartog CS. What matters most to sepsis survivors: a qualitative analysis to identify specific health-related quality of life domains. Qual Life Res. 2019;28:637-647. doi:10.1007/s11136-018-2028-8

28. Perner A, Rhodes A, Venkatesh B, et al. Sepsis: frontiers in supportive care, organisation and research. Intensive Care Med. 2017;43:496-508. doi:10.1007/s00134-017-4677-4

29. Larsen FF, Petersen JA. Novel biomarkers for sepsis: a narrative review. Eur J Intern Med. 2017;45:46-50. doi:10.1016/j.ejim.2017.09.030

30. Rello J, van Engelen TSR, Alp E, et al. Towards precision medicine in sepsis: a position paper from the European Society of Clinical Microbiology and Infectious Diseases. Clin Microbiol Infect. 2018;24:1264-1272. doi:10.1016/j.cmi.2018.03.011

31. Ritchie MD, Moore JH, Kim JH. Translational bioinformatics: biobanks in the precision medicine era. Pac Symp Biocomput. 2020;25:743-747.

32. Stanski NL, Wong HR. Prognostic and predictive enrichment in sepsis. Nat Rev Nephrol. 2020;16:20-31. doi:10.1038/s41581-019-0199-3

33. Jr RB, Hassan U, Seymour C, et al. Point-of-care sensors for the management of sepsis. Nat Biomed Eng. 2018;2:640-648. doi:10.1038/s41551-018-0288-9

34. Lopez-Campos G, Lopez-Alonso V, Martin-Sanchez F. Training health professionals in bioinformatics. Experiences and lessons learned. Methods Inf Med. 2010;49:299-304. doi:10.3414/ME09-02-0008

35. Leopold SJ, Ghose A, Plewes KA, et al. Point-of-care lung ultrasound for the detection of pulmonary manifestations of malaria and sepsis: an observational study. PLoS One. 2018;13:e0204832. doi:10.1371/journal.pone. 0204832

36. Lima A, van Rooij T, Ergin B, et al. Dynamic contrast-enhanced ultrasound identifies microcirculatory alterations in sepsis-induced acute kidney injury. Crit Care Med. 2018;46:1284-1292. doi:10.1097/CCM.0000000000003209

37. Shrestha GS, Srinivasan S. Role of point-of-care ultrasonography for the management of sepsis and septic shock. Rev Recent Clin Trials. 2018;13:243-251. doi:10.2174/1574887113666180412165405

38. Cawcutt KA, Peters SG. Severe sepsis and septic shock: clinical overview and update on management. Mayo Clin Proc. 2014;89:1572-1578. doi:10.1016/j.mayocp.2014.07.009

39. Henwood PC, Mackenzie DC, Rempell JS, et al. A practical guide to self-sustaining point-of-care ultrasound education programs in resource-limited settings. Ann Emerg Med. 2014;64:277-285.e2. doi:10.1016/j.annemergmed.2014.04.013

40. Swamy L, Worsham C, Bialas MJ, et al. The 60-minute root cause analysis: a workshop to engage interdisciplinary clinicians in quality improvement. MedEdPORTAL. 2018;14:10685. doi:10.15766/ mep_2374-8265.10685 


\section{Publish your work in this journal}

Therapeutics and Clinical Risk Management is an international, peerreviewed journal of clinical therapeutics and risk management, focusing on concise rapid reporting of clinical studies in all therapeutic areas outcomes, safety, and programs for the effective, safe, and sustained use of medicines. This journal is indexed on PubMed Central, CAS,
EMBase, Scopus and the Elsevier Bibliographic databases. The manuscript management system is completely online and includes a very quick and fair peer-review system, which is all easy to use. Visit http://www.dovepress.com/testimonials.php to read real quotes from published authors. 